\title{
Confidence interval for number of population in dynamical stochastic exponential population growth models
}

\author{
Morteza Khodabin*, Mohammad Ahmad Naeini \\ Department of Mathematics, Karaj Branch, Islamic Azad University, Karaj, Iran \\ Department of Computer Engineering, Karaj Branch, Islamic Azad University, Karaj, Iran \\ *Corresponding author E-mail: m-khodabin@kiau.ac.ir
}

\begin{abstract}
In this paper, the confidence interval for the solution of stochastic exponential population growth model where the so-called parameter, population growth rate is not completely definite and it depends on some random environmental effects is obtained. We use Iran population data in the period 1921-2006 as an example.
\end{abstract}

Keywords: Brownian motion process; Ito integral; Exponential growth model; Confidence interval.

\section{Introduction}

Population growth models are abstract representation of the real world objects, systems or processes to illustrate the theoretical concepts that in these days are increasingly being used in more applied situations such as predicting future outcomes or simulation experimentation. In mathematical literature, many population models have been considered, from deterministic and stochastic population models where the population size is represented by a discrete random variable, to very complex continuous stochastic models. A nonrandom case, ignore natural variation and produce a single value result, while a stochastic model incorporates some natural variation in to model to state unpredictable situations such as weather or random fluctuations in resources and will generate a mean or most probable result. Nowadays, the well-known model like logistic play a major role in modern ecological theory.

The paper [2], introduce numerical approximations for population growth models. In paper [3], the stochastic and generalized stochastic exponential population growth models are introduced. So, in the present paper, We construct a confidence interval for number of population obtained in [3].

\section{Stochastic concepts and Ito integral}

Definition 2.1 (Brownian motion process). Brownian motion B(t) is a stochastic process with the following properties.

(i) (Independence of increments) $B(t)-B(s)$, for $t>s$, is independent of the past.

(ii) (Normal increments) $B(t)-B(s)$ has Normal distribution with mean 0 and variance $t-s$. This implies (taking $s=0)$ that $B(t)-B(0)$ has $N(0, t)$ distribution.

(iii) (Continuity of paths) $B(t), t \geq 0$ are continuous functions of $t$.

Definition 2.2 Let $\{N(t)\}_{t \geq 0}$ be an increasing family of $\sigma$-algebras of sub-sets of $\Omega$. A process $g(t, \omega)$ from $[0, \infty) \times$ $\Omega$ to $R^{n}$ is called $N(t)$-adapted if for each $t \geq 0$ the function $\omega \longrightarrow g(t, \omega)$ is $N(t)$-measurable.

Definition 2.3 Let $\nu=\nu(S, T)$ be the class of functions $f(t, \omega):[0, \infty) \times \Omega \longrightarrow R$ such that, on $\Omega$.

(i) $(t, \omega) \longrightarrow f(t, \omega)$, is $B \times \mathcal{F}$-measurable, where $B$ denotes the Borel $\sigma$-algebra on $[0, \infty)$ and $\mathcal{F}$ is the $\sigma$-algebra

(ii) $f(t, \omega)$ is $\mathcal{F}_{t}$-adapted, where $\mathcal{F}_{t}$ is the $\sigma$-algebra generated by the random variables $B(s) ; s \leq t$. 
(iii) $E\left[\int_{S}^{T} f^{2}(t, \omega) d t\right]<\infty$.

Definition 2.4 (The Itô integral), [1]. Let $f \in \nu(S, T)$, then the Itô integral of $f$ (from $S$ to $T$ ) is defined by

$$
\int_{S}^{T} f(t, \omega) d B(t)(\omega)=\lim _{n \rightarrow \infty} \int_{S}^{T} \phi_{n}(t, \omega) d B(t)(\omega), \quad\left(\text { limit } \quad \text { in } \quad L^{2}(P)\right)
$$

where, $\phi_{n}$ is a sequence of elementary functions such that

$$
E\left[\int_{S}^{T}\left(f(t, \omega)-\phi_{n}(t, \omega)\right)^{2} d t\right] \rightarrow 0, \quad \text { as } \quad n \rightarrow \infty .
$$

Theorem 2.5 (The Itô isometry). Let $f \in \nu(S, T)$, then

$$
E\left[\left(\int_{S}^{T} f(t, \omega) d B(t)(\omega)\right)^{2}\right]=E\left[\int_{S}^{T} f^{2}(t, \omega) d t\right] .
$$

Proof. see [1].

Definition 2.6 (1-dimensional Itô processes), [1]. Let $B(t)$ be 1-dimensional Brownian motion on $(\Omega, \mathcal{F}, P)$. $A$ 1 -dimensional Itô process (stochastic integral) is a stochastic process $X(t)$ on $(\Omega, \mathcal{F}, P)$ of the form

$$
X(t)=X(0)+\int_{0}^{t} u(s, \omega) d s+\int_{0}^{t} v(s, \omega) d B(s),
$$

or

$$
d X(t)=u d t+v d B(t)
$$

where

$$
\begin{gathered}
P\left[\int_{0}^{t} v^{2}(s, \omega) d s<\infty, \quad \text { for } \quad \text { all } \quad t \geq 0\right]=1, \\
P\left[\int_{0}^{t}|u(s, \omega)| d s<\infty, \quad \text { for } \quad \text { all } \quad t \geq 0\right]=1 .
\end{gathered}
$$

Theorem 2.7 (The 1-dimensional Itô formula). Let $X(t)$ be an Itô process given by (1) and $g(t, x) \in C^{2}([0, \infty) \times$ $R)$, then

is again an Itô process, and

$$
Y(t)=g(t, X(t))
$$

$$
\begin{gathered}
d Y(t)=\frac{\partial g}{\partial t}(t, X(t)) d t+\frac{\partial g}{\partial x}(t, X(t)) d X(t)+\frac{1}{2} \frac{\partial^{2} g}{\partial x^{2}}(t, X(t))(d X(t))^{2}, \\
\text { where }(d X(t))^{2}=(d X(t))(d X(t)) \text { is computed according to the rules } \\
d t . d t=d t . d B(t)=d B(t) . d t=0, \quad d B(t) . d B(t)=d t .
\end{gathered}
$$

Proof. see [1].

\section{$3 \quad$ Exponential population growth models}

Exponential model as a J-shaped process, refers to continuous population growth in an unlimited environment which is appropriate for populations with overlapping generations. In deterministic cases, we assume that $a(t)=r(t)$ is an accurate and nonrandom given function whereas in stochastic forms, $a(t)$ at time $t$ is not completely definite and it depends on some random environment effects, i.e.

$$
a(t)=r(t)+" \text { noise" }
$$

, where $r(t)$ is a nonrandom function of time variable that means the growth rate of population at time $t$ whereas we do not know the exact behavior of "noise" term, we can set,

$$
a(t)=r(t)+\alpha(t) W(t),
$$

where $W(t)=\frac{d B(t)}{d t}$ is one dimensional white noise process and $B(t)$ is a one dimensional brownian motion and $\alpha(t)$ is nonrandom function that shows the infirmity and intensity of noise at time $t$. 


\subsection{Dynamical deterministic exponential population growth model}

Consider the following simple population growth model

$$
\frac{d N(t)}{d t}=r(t) N(t)
$$

It's solution is

$$
N(t)=N(0) \exp \left(\int_{0}^{t} r(s) d s\right)
$$

if $r(t)=r$, then

$$
N(t)=N(0) e^{r t} .
$$

\subsection{Dynamical stochastic exponential population growth model}

The stochastic exponential model is as follows

$$
\frac{d N(t)}{d t}=\left(r(t)+\alpha(t) \frac{d B(t)}{d t}\right) N(t)
$$

then

$$
d N(t)=(r(t) d t+\alpha(t) d B(t)) N(t),
$$

we can find that $[3]$

$$
N(t)=N(0) \exp \left(\int_{0}^{t}\left(r(s)-\frac{1}{2} \alpha^{2}(s)\right) d s+\int_{0}^{t} \alpha(s) d B(s)\right)
$$

\section{Confidence interval}

Since $N(t)$ is a random process, we can construct an confidence interval for it.

Theorem 4.1 Let $\alpha(t)$ be non-random such that $\int_{0}^{t} \alpha^{2}(s) d s<\infty$, then $(1-\epsilon) \%$ confidence interval for $N(t)$ is given by

$$
N(t)=N(0) \exp \left(\int_{0}^{t}\left(r(s)-\frac{1}{2} \alpha^{2}(s)\right) d s\right) \exp \left( \pm Z_{\frac{\epsilon}{2}} \sqrt{\left.\int_{0}^{t} \alpha^{2}(s) d s\right)}\right.
$$

proof:

It is easy to see that if $\alpha(t)$ is non-random such that $\int_{0}^{t} \alpha^{2}(s) d s<\infty$, then its Itô integral $Y(t)=\int_{0}^{t} \alpha(s) d B(s)$ is a Gaussian process with zero mean and variance given by $\int_{0}^{t} \alpha^{2}(s) d s$. So we can rewrite $(9)$ as

$$
N(t)=N(0) \exp \left(\int_{0}^{t}\left(r(s)-\frac{1}{2} \alpha^{2}(s)\right) d s\right) \exp \left(\int_{0}^{t} \alpha(s) d B(s)\right)=D(t) \exp \left(\int_{0}^{t} \alpha(s) d B(s)\right),
$$

where

$$
D(t)=N(0) \exp \left(\int_{0}^{t}\left(r(s)-\frac{1}{2} \alpha^{2}(s)\right) d s\right) .
$$

Thus

$$
\int_{0}^{t} \alpha(s) d B(s)=\ln \frac{N(t)}{D(t)} \sim N\left(0, \int_{0}^{t} \alpha^{2}(s) d s\right)
$$

so we can put

$$
-Z_{\frac{\epsilon}{2}} \sqrt{\int_{0}^{t} \alpha^{2}(s) d s} \leq \ln \frac{N(t)}{D(t)} \leq Z_{\frac{\epsilon}{2}} \sqrt{\int_{0}^{t} \alpha^{2}(s) d s}
$$

or

$$
D(t) \exp \left(-Z_{\frac{\epsilon}{2}} \sqrt{\left.\int_{0}^{t} \alpha^{2}(s) d s\right)} \leq N(t) \leq D(t) \exp \left(Z_{\frac{\epsilon}{2}} \sqrt{\left.\int_{0}^{t} \alpha^{2}(s) d s\right)}\right.\right.
$$


Table 1: Number of population and population growth rate (Iran).

\begin{tabular}{ccc}
\hline Year & $N(t)$ & $r(t)$ \\
\hline 1300 & 9707000 & --- \\
1305 & 10456000 & 0.014976785 \\
1310 & 11185000 & 0.014273539 \\
1315 & 11964000 & 0.014034563 \\
1320 & 12833000 & 0.014056511 \\
1325 & 14159000 & 0.01521471 \\
1330 & 16237000 & 0.017296051 \\
1335 & 18954704 & 0.019304099 \\
1345 & 25788722 & 0.021950555 \\
1355 & 33708744 & 0.022892836 \\
1365 & 49445010 & 0.025362662 \\
1370 & 55837163 & 0.025309149 \\
1375 & 60055488 & 0.02459658 \\
1385 & 70495782 & 0.023600125 \\
\hline
\end{tabular}

which is equal to $(10)$.

In special case, if $r(t)=r$ and $\alpha(t)=\alpha$, we can write (9) as

$$
N(t)=N(0) \exp \left(\left(r-\frac{1}{2} \alpha^{2}\right) t+\alpha B(t)\right),
$$

and (10) as

$$
N(0) \exp \left(\left(r-\frac{1}{2} \alpha^{2}\right) t-Z_{\frac{\epsilon}{2}}|\alpha| \sqrt{t}\right) \leq N(t) \leq N(0) \exp \left(\left(r-\frac{1}{2} \alpha^{2}\right) t+Z_{\frac{\epsilon}{2}}|\alpha| \sqrt{t}\right) .
$$

\section{Numerical example}

The following table represent the number of population of Iran in the period 1300-1385. In table 1, we assume that $\mathrm{t}=0$ correspond to 1300 , and so the initial population is $N(0)=9707000$. We compute the population growth rate $r(t)$ by the following relation

$$
r(t)=\sqrt[t]{\frac{N(t)}{N(0)}}-1
$$

The scatter plot of $r(t)$ shows that we can fit multiple linear regression for $r(t)$ as below

$$
\hat{r}(t)=0.0153-0.000267 t+0.000013 t^{2}-0.000000104 t^{3}, \quad t \geq 0 .
$$

with $R$ squared $=0.982$. Here we assume that $\alpha(s)=\frac{1}{15} \sqrt{\frac{1}{1+s}}$.

So for example if we put $t=90$, we get

$\int_{0}^{90} \hat{r}(s) d s=1.74879, \int_{0}^{90} \frac{\alpha^{2}(s)}{2} d s=0.010024, \int_{0}^{90} \alpha^{2}(s) d s=0.020048, \int_{0}^{90} \alpha(s) d B(s)=0.1457$.

By using above outputs and from (9) the prediction of population at time $t$ will be

$$
\hat{N}(90)=9707000 \times e^{1.74879-0.010024+0.1457}=63901144.67 .
$$

Consequently, the 95 percent confidence interval for $N(t)$ would be

$$
41864460.75 \leq N(t) \leq 72881677.85
$$




\section{References}

[1] B. Oksendal, Stochastic Differential Equations, An Introduction with Applications, Fifth Edition, Springer-Verlag, New York, (1998).

[2] Kamel Al-Khaled, Numerical approximations for population growth models, Applied Mathematics and Computation 160 (2005) 865-873.

[3] khodabin, M., Maleknejad, K., Rostami, M., Nouri,M. Interpolation solution in generalized stochastic exponential population growth model, Mathematical and Computer Modelling, 53: 1910-1920. (2011). 